\title{
Progressive Failure Analysis Method of a Pi Joint with Uncertainties in Fracture Properties
}

\author{
Wooseok $\mathrm{Ji}^{1}$ and Anthony M. Waas ${ }^{2}$ \\ University of Michigan, Ann Arbor, MI, 48109 \\ and \\ Ravi S. Raveendra ${ }^{3}$ \\ Comet Technology Corporation, Ann Arbor, MI, 48103
}

\begin{abstract}
In this presentation, the structural performance of fiber reinforced composite laminate $\mathrm{Pi}$ joints is studied through a progressive failure analysis (PFA) method. The bonded joint area that is the weakest link of the Pi joint structure is modeled using the discrete cohesive zone method (DCZM). The damage growth and failure of the bonded interface is modeled through an exponential decaying traction-separation law that governs the behavior of DCZM elements. This interface model is implemented into a non-linear finite element (FE) code for modeling the progressive failure of the composite Pi joint structures. The present PFA framework is incorporated with a probabilistic analysis module to consider material variability and manufacturing inconsistencies. The proposed PFA methodology is demonstrated for a 2D Pi-shaped laminate composite structure adhesively bonded through a Pi joint, and subjected to a pull-off load.
\end{abstract}

\section{Nomenclature}

$\begin{array}{ll}G_{I C} & =\text { Mode I critical strain energy release rate } \\ G_{I I C} & =\text { Mode II critical strain energy release rate } \\ \sigma_{C} & =\text { critical cohesive strength of Mode I } \\ \tau_{C} & =\text { critical cohesive strength of Mode II } \\ \delta_{i C} & =\text { critical relative displacement fields }(i=1 \text { and } 2) \\ \delta_{i} & =\text { relative displacement fields }(i=1 \text { and } 2) \\ \alpha_{i} & =\text { softening rate of a traction separation law }(i=1 \text { and } 2) \\ \widetilde{K}_{i}^{(m n)} & =\text { initial stiffness of a DCZM subelement }\end{array}$

\section{Introduction}

\begin{abstract}
dhesively bonded joint technology is now widely used in aircraft structural designs because of its advantage Aover conventional fastening systems. Stress concentrations that are unavoidable at fastener areas can be reduced with adhesively bonded joints, and thus fatigue resistance can be significantly improved. Structural weight can be reduced by replacement of the fastener hardware with the adhesive joints. Various adhesive joint profiles are available for joining structural parts in a complex configuration that may be difficult and costly to achieve with a mechanical fastening system. Especially, an emerging and promising concept in joining laminated structures is the "Pi joint". The Pi-shaped joint improves performance by increasing the bonding area between adherends. However, the bonded interface is still the weakest link due to the large amount of load being transmitted over the region, and thus interfacial failure is the major concern in such laminated composites.
\end{abstract}

\footnotetext{
${ }^{1}$ Research Fellow, Department of Aerospace Engineering, 1320 Beal Avenue, Ann Arbor, MI 48109.

${ }^{2}$ Felix Pawlowski Collegiate Professor, Department of Aerospace Engineering, 1320 Beal Avenue, Ann Arbor, MI 48109, AIAA fellow.

${ }^{3}$ President, 3830 Packard Road, Ann Arbor, MI 48103.
} 
Locations of delamination onset and their evolution processes vary, and they are highly dependent on loading types causing separation at the bonding interfaces. For composite structures in aerospace applications, impact loading due to foreign objects can leave a local initial disbond at the joint interface, which may be difficult to detect without a special non-destructive evaluation (NDE) technique. The local failure may grow from the initial size and result in continued degradation of structural performance, leading to the collapse of entire structures. Of interest is a quantitative characterization of the strength and stiffness of the joint in terms of interface fracture properties, joint geometry, material properties of the joint and initial disbond. Uncertainties in bond quality and the occurrence of initial disbonds caused by impact or unintentionally introduced at the manufacturing stage can result in a joint that has unsatisfactory structural performance. In order to improve structural integrity and damage tolerance (SIDT), the joint model needs to be assumed to have defects in bonding interfaces. It will be desirable to characterize the joint performance using probabilistic-based analysis to account for these uncertainties in geometrical and material properties.

In this paper, results of a probabilistic finite element analysis on the performance of a Pi-joint are presented accounting for variability in the bonding quality of the joint. It is assumed that the joint has no initial disbond at the interfaces, but has manufacturing inconsistencies in the fracture properties of the adhesive layer. Damage growth and failure of adhesive joints is modeled using the discrete cohesive zone model (DCZM) developed by ideas first introduced by Song and Waas ${ }^{1}$ and further refined by Xie and Waas ${ }^{2}$ and Gustafson and Waas ${ }^{3}$. The DCZM employed here has been applied to various engineering problems and proved to be an efficient and accurate tool for predicting discontinuous surface behaviors prescribed by a traction-displacement separation law ${ }^{1-4}$. The DCZM is implemented as user elements into the commercial finite element analysis package, ABAQUS, through a user subroutine. Incorporation of the DCZM elements into the conventional finite element (FE) framework implies that various failure modes (material failure, crack propagation, and local buckling) are tracked simultaneously, thus any potential interaction between the failure modes can be captured.

\section{DCZM formulation with an exponential softening traction-separation law}

The two-dimensional (2D) DCZM elements with an exponential softening traction-separation law are utilized in this study to predict delamination initiation and its growth at joint interfaces. Figure 1 illustrates the initial configuration of a DCZM element in the reference $X Y$ coordinate system, and the deformed configuration with the local $x y$-axis. The DCZM element consists of nonlinear 1D subelements connecting the adjoining nodes, which have initially zero separation. The configuration of the DCZM element conforms to a typical four-node quadrilateral element as shown in Figure 1. However, in operation, it is discrete in nature since the delamination progress at the crack tip is discretized with the successive failure of the non-linear 1D decohering subelements. Typically, delamination growth modes are categorized as Mode I (opening), Mode II (in-plane shear), and Mode III (out-ofplane shear), dependent on the crack tip deformation state. Usually, loading and boundary conditions of composite structures in aerospace applications are complex, and thus the delamination process is, in general, of the mixedmode type. The subelement behavior is decomposed into two components in the local Mode I and Mode II directions, and each mode is governed by its own traction-separation law.
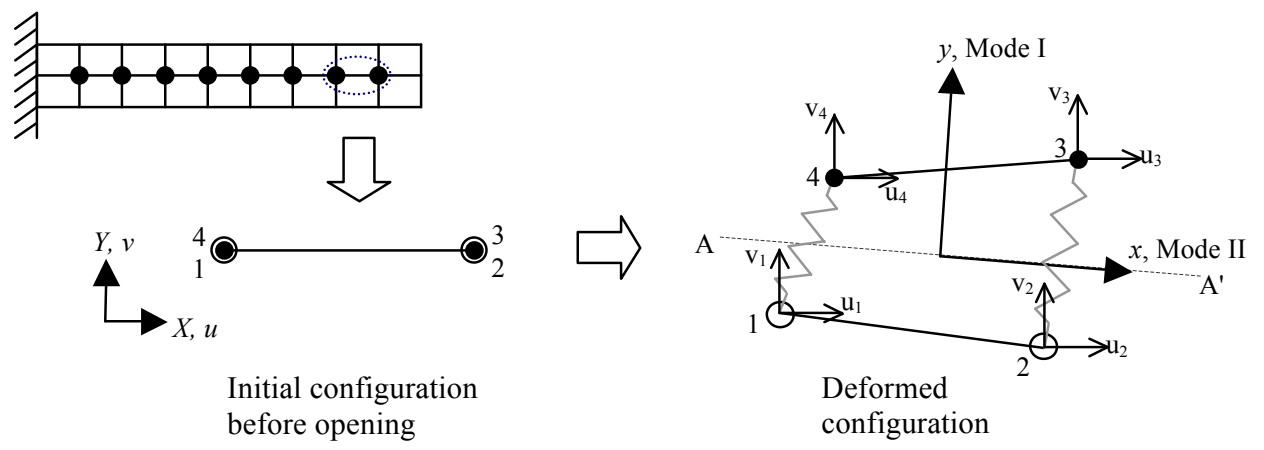

Figure 1. Typical FE model of a double cantilever beam with DCZM elements inserted at the interface. Initial and deformed configurations of a DCZM element are illustrated.

The damage and failure behavior of the non-linear 1D decohesion element is modeled through a tractionseparation law, where the strength of the subelement is expressed as a function of the relative displacement field for each degree of freedom (DOF). Individual traction-separation laws are assigned to each DOF of the subelement to 
model the mixed fracture behavior at the interface. Thus, the DCZM is the decohesive analog of the virtual crack closure technique (VCCT) introduced by Kanninen and Rybicki ${ }^{5}$. Figure 2 shows the exponential softening tractionseparation laws for (a) Mode I and (b) Mode II fracture behaviors, respectively. The fracture energy ${ }^{6}$ and cohesive strengths for each mode are the two key parameters that characterize the traction separation laws. The area under the curve corresponds to the fracture energy $\left(G_{I C}\right.$ or $\left.G_{I I C}\right)$ and the peak of the curve is associated with the cohesive strength $\left(\sigma_{C}\right.$ or $\left.\tau_{C}\right)$ of the adhesive material. Thus, local elemental stiffness matrix and force vector for the DCZM elements can be expressed with the two parameters when the current relative displacement fields are known.
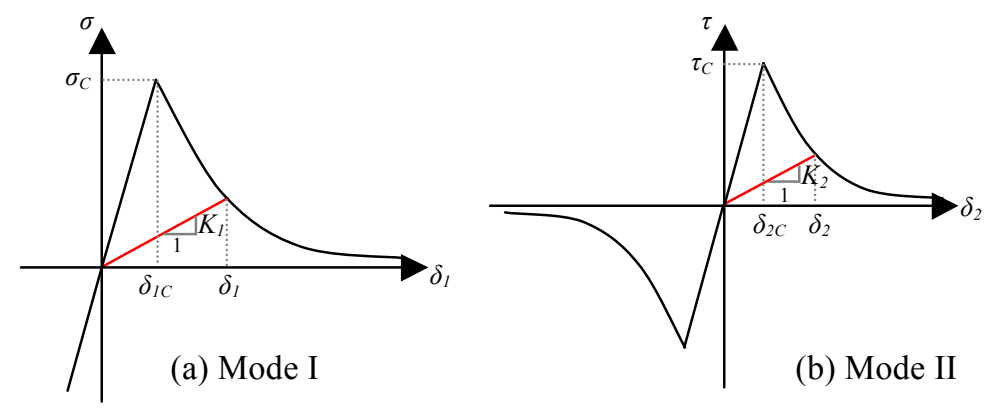

Figure 2. Exponential softening traction-separation law for (a) Mode I and (b) Mode II fracture behavior. Linear elastic behavior is assumed before the DCZM subelements are damaged. The stiffness reduction of the DCZM elements is naturally calculated from these traction-separation laws.

At every deformed state during explicit incremental or implicit iterative analysis within a global FE framework, the local $x y$-axis of each DCZM element needs to be first defined to obtain relative displacements in directions of each fracture mode. The relative displacements are then used to calculate the forces from the traction-separation laws. Once the current displacement fields and coordinate information at the four nodes are passed in, DCZM algorithm first sets up its local coordinate system whose $x$-axis is aligned along the line connecting the 1D element midpoints as shown in Figure 1. The Mode I direction now conforms to the local $y$-axis and the Mode II direction is aligned with the local $x$-axis. The global displacement fields, $u_{i}$ and $v_{i}$, are then rotated to the local coordinate system through the scalar projection to base vectors of the local axis. When the relative displacement fields associated with each fracture modes are obtained, the corresponding forces can be calculated from the traction-separation laws. Let $\delta_{1}$ and $\delta_{2}$ be the current relative displacement fields with respect to the local $x y$-axis where $i=1$ and 2 for Mode I and II respectively, then the force for Mode I is obtained as

$$
F_{1}^{(m n)}=\left\{\begin{array}{l}
\Delta a \widetilde{K}_{1}^{(m n)} \delta_{1}^{(m n)} \text { if } \delta_{1}^{(m n)} \leq \delta_{1 C}^{(m n)} \\
\Delta a \widetilde{K}_{1}^{(m n)} \delta_{1 C}^{(m n)} \exp \left[\alpha_{1}\left(1-\frac{\delta_{1}^{(m n)}}{\delta_{1 C}^{(m n)}}\right)\right] \text { if } \delta_{1}^{(m n)}>\delta_{1 C}^{(m n)}
\end{array}\right.
$$

and for Mode II,

$$
F_{2}^{(m n)}=\left\{\begin{array}{l}
\Delta a \widetilde{K}_{2}^{(m n)} \delta_{2}^{(m n)} \text { if }\left|\delta_{2}^{(m n)}\right| \leq \delta_{2 C}^{(m n)} \\
\Delta a \widetilde{K}_{2}^{(m n)} \delta_{2 C}^{(m n)} \exp \left[\alpha_{2}\left(1-\frac{\delta_{2}^{(m n)}}{\delta_{2 C}^{(m n)}}\right)\right] \text { if }\left|\delta_{2}^{(m n)}\right|>\delta_{2 C}^{(m n)}
\end{array}\right.
$$

where $\widetilde{K}_{i}^{(m n)}$ is the initial stiffnesses of the subelements in the Mode I and II directions, respectively, and $\Delta a$ is the effective area where the element force is applied. Note that the effective area $\Delta a$ depends on the element size, $l_{\mathrm{el}}$. The superscript $m$ and $n$ denote the adjoining node numbers (1-4 and 2-3). In Figure 1, the $y$-axis divides the elements into two regions and each region is defined as the effective area for each subelement. The value of the initial stiffnesses should be high enough to avoid adding artificial compliance to the model, but not so high as to cause numerical instability. Xie and Waas ${ }^{1}$ suggested that $\widetilde{K}_{i}^{(m n)}$ be three orders of magnitude larger than the major stiffness of the body material. For an adhesive layer, they proposed that $\widetilde{K}_{i}^{(m n)}$ be estimated from the adhesive properties, based on the equivalence: 


$$
\widetilde{K}_{1}^{(m n)}=\frac{E B l_{\mathrm{el}}}{h} \text { and } \widetilde{K}_{2}^{(m n)}=\frac{G B l_{\mathrm{el}}}{h}
$$

where $E$ and $G$ are the Young's modulus and shear modulus of the adhesive layer, respectively, $B$ is the out-of-plane thickness of the body, $h$ is the thickness of the adhesive layer, and $l_{\mathrm{el}}$ is the element size. The critical displacements, $\delta_{i C}$, can be obtained from

$$
\begin{aligned}
& \widetilde{K}_{1}^{(m n)} \delta_{1 C}=\sigma_{C} \\
& \widetilde{K}_{2}^{(m n)} \delta_{2 C}=\tau_{C}
\end{aligned}
$$

and thus the critical displacements are

$$
\begin{gathered}
\delta_{1 C}=\frac{\sigma_{C}}{\widetilde{K}_{1}^{(m n)}} \\
\delta_{2 C}=\frac{\tau_{C}}{\widetilde{K}_{2}^{(m n)}}
\end{gathered}
$$

The softening rate parameter, $\alpha_{i}$, can be obtained from the area under the curve that corresponds to the fracture energy. From integrating the traction-separation laws for $\delta_{i} \in[0, \infty]$, the critical strain release rates are

$$
\begin{aligned}
G_{I C} & =\int_{0}^{\infty} \frac{F_{1}^{(m n)}}{\Delta a} \mathrm{~d} \delta_{1} \\
G_{I I C} & =\int_{0}^{\infty} \frac{F_{2}^{(m n)}}{\Delta a} \mathrm{~d} \delta_{2}
\end{aligned}
$$

and $\alpha_{i}$ can be determined as

$$
\begin{aligned}
& \alpha_{1}=\frac{\sigma_{C}^{2}}{2 G_{I C} \widetilde{K}_{1}^{(m n)}-\sigma_{C}^{2}} \\
& \alpha_{2}=\frac{\tau_{C}^{2}}{2 G_{I I C} \widetilde{K}_{2}^{(m n)}-\tau_{C}^{2}}
\end{aligned}
$$

Thus, the total force vector for the DCZM element is now fully defined as,

with the displacement vector given as,

$$
\mathbf{F}_{\mathrm{el}}=\left\{-F_{1}^{(14)},-F_{2}^{(14)}-F_{1}^{(23)},-F_{2}^{(23)}, F_{1}^{(23)}, F_{2}^{(23)}, F_{1}^{(14)}, F_{2}^{(14)}\right\}^{T}
$$

$$
\mathbf{u}_{\mathrm{el}}=\left\{u_{1}^{\prime}, v_{1}^{\prime}, u_{2}^{\prime}, v_{2}^{\prime}, u_{3}^{\prime}, v_{3}^{\prime}, u_{4}^{\prime}, v_{4}^{\prime}\right\}^{T}
$$

where the superscript 'prime' indicates that the displacement fields are written with respect to the local $x y$-axis. The corresponding stiffness matrix for the DCZM element is obtained as,

$$
\mathbf{K}_{e l}=-\frac{\mathrm{d} \mathbf{F}_{e l}}{\mathrm{~d} \mathbf{u}_{e l}}
$$

The stiffness matrix in the full matrix form ${ }^{3}$ is written as

$$
\mathbf{K}_{e l}=\left[\begin{array}{cccccccc}
K_{1}^{(14)} & 0 & 0 & 0 & 0 & 0 & -K_{1}^{(14)} & 0 \\
0 & K_{2}^{(14)} & 0 & 0 & 0 & 0 & 0 & -K_{2}^{(14)} \\
0 & 0 & K_{1}^{(23)} & 0 & -K_{1}^{(23)} & 0 & 0 & 0 \\
0 & 0 & 0 & K_{2}^{(23)} & 0 & 0 & 0 & 0 \\
0 & 0 & -K_{1}^{(23)} & 0 & K_{1}^{(23)} & 0 & 0 & 0 \\
0 & 0 & 0 & -K_{2}^{(23)} & 0 & K_{2}^{(23)} & 0 & 0 \\
-K_{1}^{(14)} & 0 & 0 & 0 & 0 & 0 & K_{1}^{(14)} & 0 \\
0 & -K_{2}^{(14)} & 0 & 0 & 0 & 0 & 0 & K_{2}^{(14)}
\end{array}\right]
$$

where the stiffness component is defined as 


$$
\begin{gathered}
K_{1}^{(m n)}=\left\{\begin{array}{l}
\Delta a \widetilde{K}_{1}^{(m n)} \text { if } \delta_{1}^{(m n)} \leq \delta_{1 C}^{(m n)} \\
-\Delta a \widetilde{K}_{1}^{(m n)} \alpha_{1} \exp \left[\alpha_{1}\left(1-\frac{\delta_{1}^{(m n)}}{\delta_{1 C}^{(m n)}}\right)\right] \quad \text { if } \delta_{1}^{(m n)}>\delta_{1 C}^{(m n)}
\end{array}\right. \\
K_{2}^{(m n)}=\left\{\begin{array}{l}
\Delta a \widetilde{K}_{2}^{(m n)} \text { if }\left|\delta_{2}^{(m n)}\right| \leq \delta_{2 C}^{(m n)} \\
-\Delta a \widetilde{K}_{2}^{(m n)} \alpha_{2} \exp \left[\alpha_{2}\left(1-\frac{\delta_{2}^{(m n)}}{\delta_{2 C}^{(m n)}}\right)\right] \text { if }\left|\delta_{2}^{(m n)}\right|>\delta_{2 C}^{(m n)}
\end{array}\right.
\end{gathered}
$$

Therefore, all the DCZM parameters including the critical separation displacements, $\delta_{i C}$, and the softening rate parameter, $\alpha_{i}$, are completely determined by the fracture properties of the cohesive layer; fracture toughness and cohesive strength. It is noted here that Eqs. (8), (9) and (11) are written with respect to the local element axis and should be rotated back to the global coordinate system before the global matrices are assembled.

\section{Structural performance of a Pi joint}

$\mathrm{Pi}$ joints are woven textile performs that have inverted Greek letter $\pi$ configurations. Composite Pi joint structures have improved load transmitting capability over conventional adhesive joint structures as shown in Figure 3. With the increased adhesive area between the skin and the adhesive joint and redistribution of stress filed throughout the pi shape, peel stress at the tips of the bonding line are significantly reduced in the Pi joints when subjected to pull-off loading. Under the pull-off loading condition, the conventional "L" shaped adhesive joints are essentially subjected to direct peel load. The conventional adhesive joints often accept bolt fastening to overcome the high peel stress issue, but the mechanical fastening system causes stress concentration at the bolted areas. It is reported that woven Pi-preform composite joints have a $250 \%$ to $300 \%$ increased load carrying capability when subjected to out-of-plane loading as compared to conventional composite joint designs.

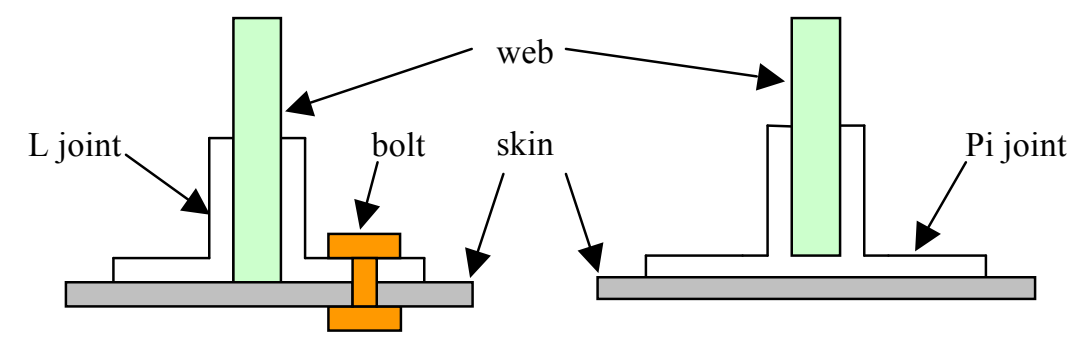

Figure 3: Conventional " $L$ " shaped joint design and Pi joint configuration.

The structural performance of the Pi joint is studied in this section using finite element analysis (FEA). Twodimensional Pi joint FE model provided from Lockheed Martin (LM) is utilized as shown in Figure 4. The Pi-joint is modeled with two-dimensional (2D) plane strain elements of ABAQUS. DCZM elements are inserted at the interface between the skin and the bottom surface of the Pi joint, and the interface between the web and the clevis in order to parametrically study the delamination failure. Adhesive layers modeled with DCZM elements are shown in red color in Figure 4. LM Pi joint uses adhesive films to bond the Pi joint to the web and skin. Fracture properties of $3 \mathrm{M}$ adhesive film, AF-191, are used for the DCZM elements ${ }^{8-10}$. The FE model is fixed at two constraint points and pulled off at the top surface of the web. Typical test setup for the pull-off loading experiments is shown in Figure 5.

Figure 6 shows a typical load-displacement curve from FEA when the DCZM elements are used to model delamination at the joint interfaces. The load is normalized by average experimental data and the displacement value at the peak load from FEA is used to normalize the displacement axis in Figure 6. At the peak load, delamination at the interface between the skin and the flange is predicted by the DCZM elements as shown in Figure 7. From the DCZM elements, the strain energy release rate for Mode II failure can be obtained along the interface and the data is used to identify the location of the failure due to the delamination. 


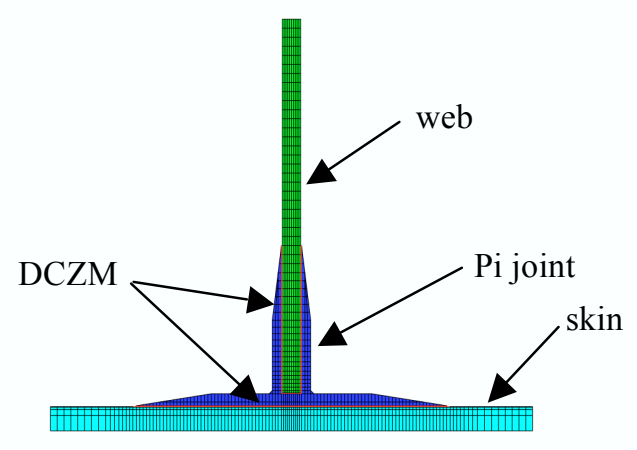

Figure 4. 2D FE model of a Pi joint structure from Lockheed Martin

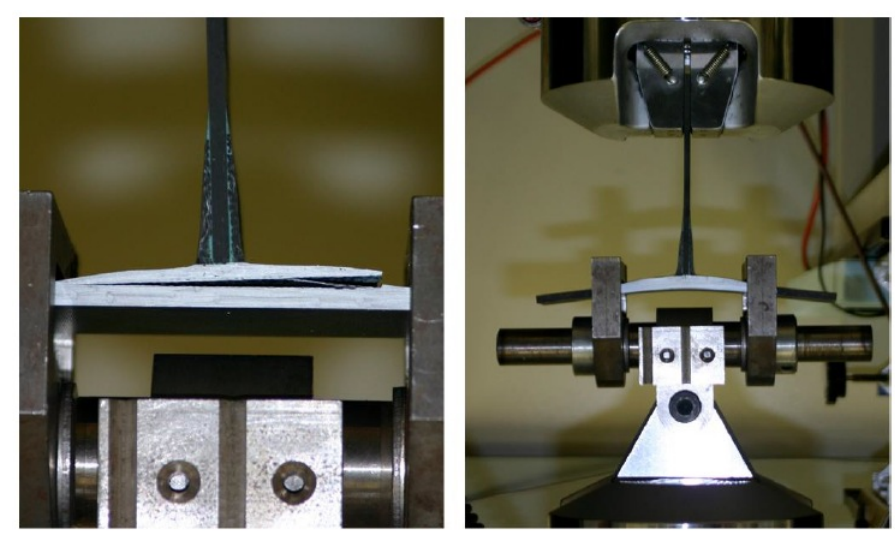

Figure 5. Typical pull-off load test setup and failure mode of Pi joint structures ${ }^{7}$

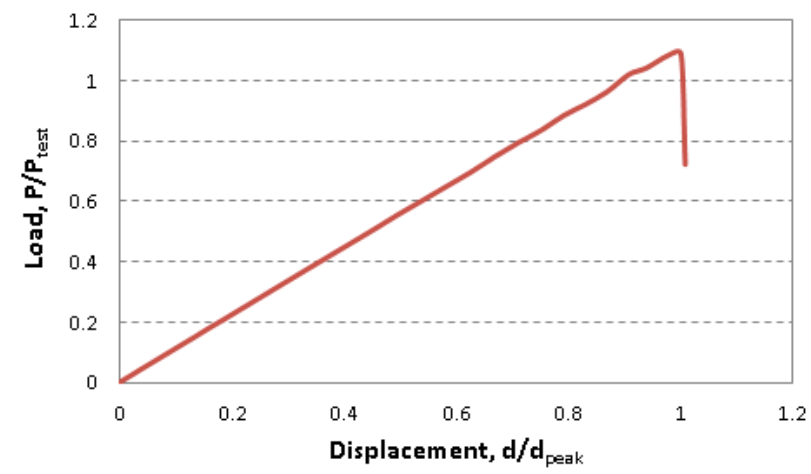

Figure 6. Typical load-displacement behavior of LM Pi joint composite

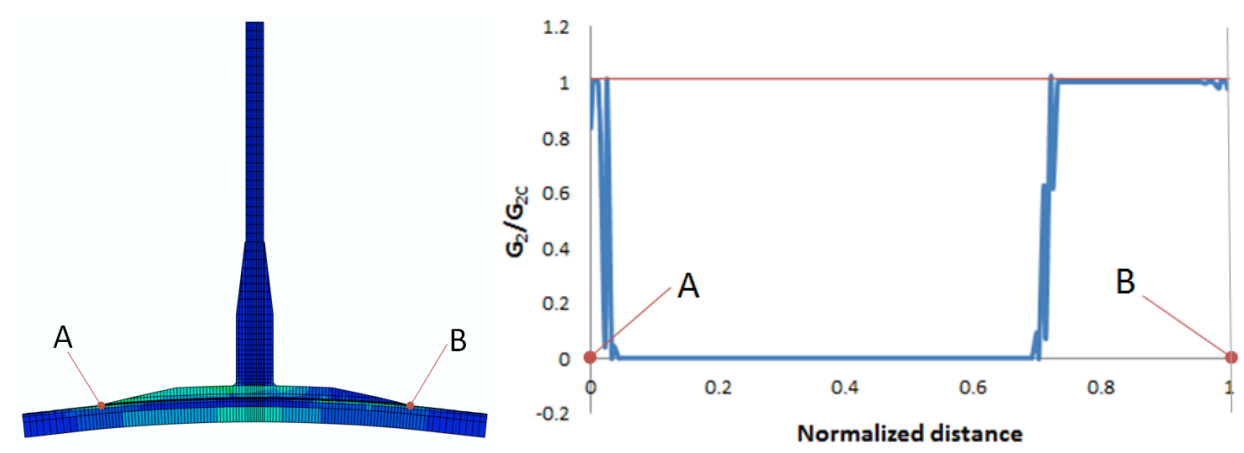

Figure 7. At the peak load, deformed configuration and strain energy release rate for Mode II along the interface between the skin and flange 
Probabilistic FEA is also performed using NESSUS software ${ }^{11,12}$. Figure 8 shows the cumulative probability of peak loads and the importance level of the parameters considered in this probabilistic FE analysis. As shown in the Figure 8 (b), the shear fracture toughness values greatly affect the performance of the Pi joint since the different deformed curvatures between the flange and skin produce large discrepancies in shear stress distribution along the bond line. This result motivates a parametric study by varying the shear fracture properties by a factor of two to examine the corresponding structural performance of Pi joint composites. Figure 9 shows the traction-separation laws when $G_{I I C}$ is varied by a factor of two. Although the exponential softening law is used in this presentation, bilinear triangular traction-separation law is used to illustrate the difference in the strain energy release rate more easily in Figure 9. Since the interface behavior between the flange and the skin is mostly governed by the adhesive paste properties and fiber bridging at the interface is non-existent, $G_{I I C}$ varies such that the three traction-separation laws have the same maximum shear displacement as shown in Figure 9.

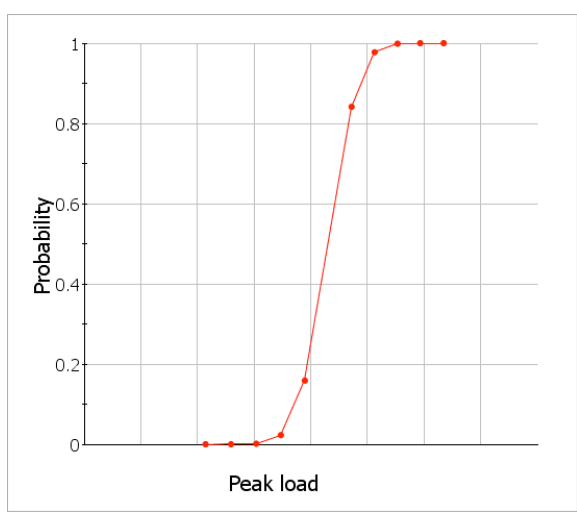

(a)

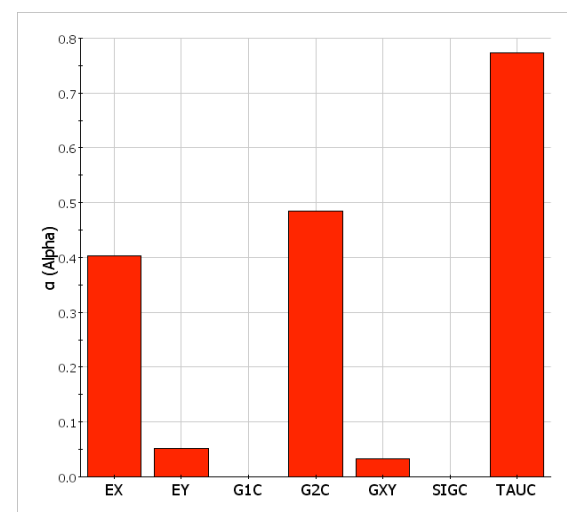

(b)

Figure 8. Probabilistic FE analysis results. (a) Cumulative distribution function of peak loads (b) Importance levels of modeling parameters.

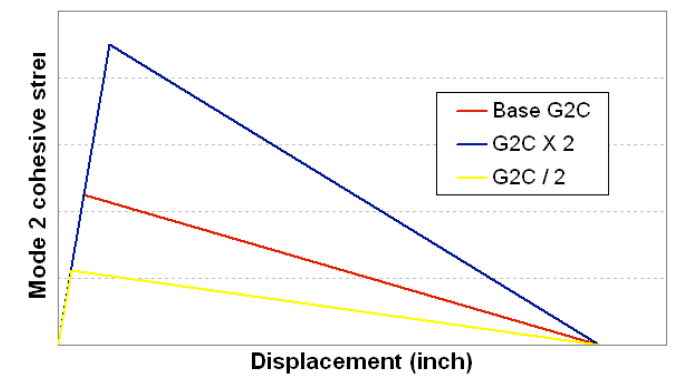

Figure 9. Traction-separation laws with a variance of the shear fracture toughness, $G_{2 C}$

For each curve in Figure 9, reliability of the Pi joint sustainability is evaluated when the variability of material properties with varying $G_{I I C}$ is considered. It is interesting to note that the weakest interface produces the most reliable Pi joint performance while the maximum peak load is found when $G_{I I C}$ is increased. With a strongly bonded interface, the response is predicted over a wide range as shown in Figure 10. It appears that the Pi joint composite structure with the lower $G_{I I C}$ consistently fails by Mode II fracture although variability in the material properties is considered. When $G_{I I C}$ is increased, making the shear toughness larger, the failure mode transitions to a mixed fracture mode that involves both Mode I and Mode II behavior, leading to the wide range of failure responses as shown in Figure 10. The importance level of each modeling parameter also indicates that the higher $G_{I I C}$ value alters the failure mode. Figure 11 shows that shear fracture properties are the most important factors defining the response of the Pi joint when the shear fracture toughness, $G_{I I C}$, is low while the Mode I behavior dominates with the higher $G_{I I C}$ values. When the resistance of the interface becomes stronger against the Mode II fracture, the failure mode is altered from Mode II to Mode I. 


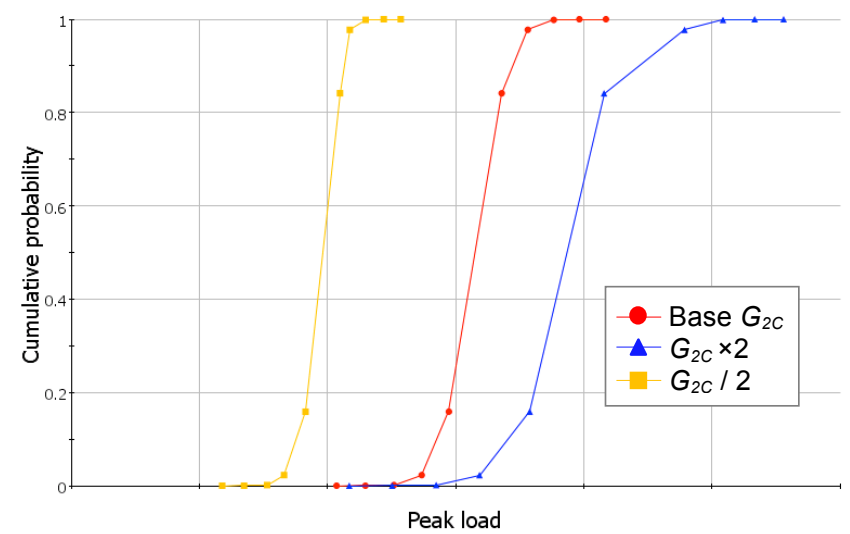

Figure 10. Probability of peak loads with a variance of the shear fracture properties

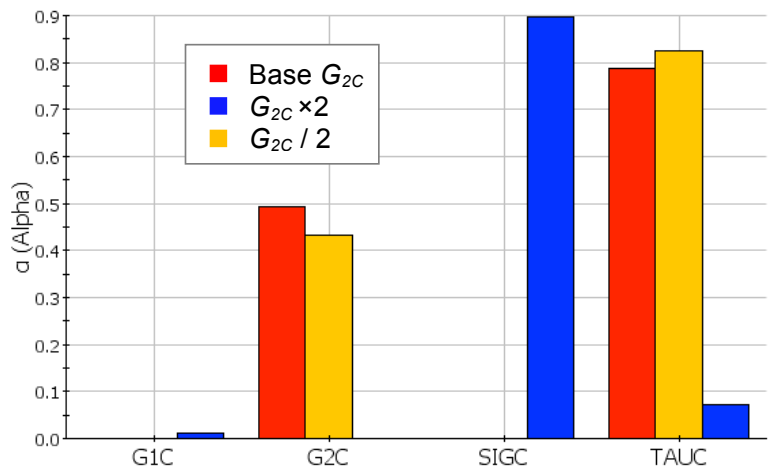

Figure 11. Importance levels of the fracture properties on each case

\section{Conclusion}

A reliability-based progressive failure analysis methodology for advanced laminated composites is demonstrated by implementing the discrete cohesive zone model for interfacial failure, coupled with a probabilistic analysis model. The discrete cohesive zone model (DCZM) elements are used to model the adhesion and delamination failure at the bonding surfaces. The PFA framework is incorporated with a probabilistic analysis module, based on the NEESUS software, to consider material variability and manufacturing inconsistencies. The methodology is applied for progressive failure analysis of a 2D Pi Joint composite structure. The PFA methodology is demonstrated for a two-dimensional (2D) Pi-shaped laminate composite structure adhesively bonded through a Pi joint, and subjected to a pull-off load. The method presented here shows that it is capable of predicting failure initiation and its growth with the capability of identifying key modeling parameters affecting the failure mechanisms.

\section{Acknowledgements}

We are grateful for financial sponsorship from the AFOSR through a STTR contract to Comet Technology Corporation. The encouragement and support of Dr. David Stargel (AFOSR) and Dr. Ed Forster (AFRL) are gratefully acknowledged.

\section{References}

\footnotetext{
${ }^{1}$ Song, S. J. and Waas, A. M., "Nonlinear elastic foundation model for crack growth in laminates," Journal of Composites Engineering, Vol. 3, No. 10, 1993, pp. 945-959.

${ }^{2}$ Xie, D. and Waas A. M., "Discrete cohesive zone model for mixed-mode fracture using finite element analysis," Engineering Fracture Mechanics, Vol. 73, No. 13, 2006, pp. 1783-1796.

${ }^{3}$ Gustafson, P. A. and Waas, A. M., "T650/AFR-PE-4/FM680-1 mode I critical energy release rate at high temperatures: Experiments and numerical models," Proceedings of the 48th AIAA/ASME/ASCE/AHS/ASC Structures, Structural Dynamics and Materials Conference, Vol. 7, 2007, pp. 7630-7377.
} 
${ }^{4}$ Gustafson, P. A., and Waas, A. M., "Efficient and robust traction laws for the modeling of adhesively bonded joints," Proceedings of the 49th AIAA/ASME/ASCE/AHS/ASC Structures, Structural Dynamics and Materials Conference, 2008.

${ }^{5}$ Rybicki, E. F. and Kanninen, M. F., "A finite element calculation of stress-intensity factors by modified crack-closure integral," Engineering Fracture Mechanics, Vol. 9, 1997, pp. 931-938.

${ }^{6}$ Bazant, Z. P., and Planas, J., "Fracture and size effect in concrete and other quasibrittle materials," CRC Press, 1998.

${ }^{7}$ Collier, C., Yarrington, P., Pickenheim M., Bednarcyk B. and Jeans J. "Analysis methods used on the NASA composite crew module (CCM)," Proceedings of the 49th AIAA/ASME/ASCE/AHS/ASC Structures, Structural Dynamics and Materials Conference, 2008.

8 Manufacturer technical datasheet, "3M Scotch-Weld Structural Adhesive Film AF 191", 2009.

9 Melcher, R. J. and Johnson W.S. "Mode I fracture toughness of an adhesively bonded composite-composite joint in a cryogenic environment," Composites Science and Technology, Vol. 67, 2007, pp. 501-506.

${ }^{10}$ Butkus, L. M., "Environmental durability of adhesively bonded joints," Ph.D. Dissertation, Mechanical Engineering Department, The Georgia Institute of Technology, Atlanta, GA, 1997.

${ }^{11}$ NESSUS Theoretical Manual, 2010.

${ }^{12}$ Thacker, B.H., Riha, D.S., Fitch, S.K., Huyse, L.J., and Pleming, J.B., "Probabilistic engineering analysis using the NESSUS software," Structural Safety, Vol. 28, No. 1-2, 2006, pp. 83-107. 